\title{
ADHOCFTSIM: A SIMULATOR OF FAULT TOLERANCE IN THE AD-HOC NETWORKS
}

\author{
Ghalem Belalem $^{1}$ and Ali Cherif Brakeche ${ }^{2}$ and Abderahmann Benaissa ${ }^{3}$ and \\ Esma Insaf Djebbar ${ }^{4}$ \\ Department of Computer Science, Faculty of Sciences, University of Oran - Algeria \\ ${ }^{1}$ ghalem1dzegmail.com \\ ${ }^{2}$ brakechedgmail.com \\ ${ }^{3}$ benaissa_infeyahoo.fr \\ ${ }^{4}$ d.insafeyahoo.fr
}

\begin{abstract}
The flexibility and diversity of Wireless Mobile Networks offer many opportunities that are not always taken into account by existing distributed systems. In particular, the proliferation of mobile users and the use of mobile Ad-Hoc promote the formation of collaborative groups to share resources. We propose a solution for the management of fault tolerance in the Ad-Hoc networks, combining the functions needed to better availability of data. Our contribution takes into account the characteristics of mobile terminals in order to reduce the consumption of resources critical that energy, and to minimize the loss of information. Our solution is based on the formation of clusters, where each is managed by a node leader. This solution is mainly composed of four sub-services, namely: prediction, replication, management of nodes in the cluster and supervision. We have shown, using several sets of simulation, that our solution is twofold: minimizing the energy consumption which increases the life of the network and better support deal with requests lost.
\end{abstract}

\section{KEYWORDS}

Ad-Hoc Network, Clustering, Replication, Energy, Critical object, Prediction.

\section{INTRODUCTION}

The advent of laptops, personal digital assistants (PDA) and powerful wireless networks (AdHoc) in the 90s pose new problems for construction of distributed systems with mobile clients. This has generated a new paradigm called "Mobile", which raises research not only to solve existing problems in distributed systems such as fault tolerance, but also to address specific problems in mobile environment such as the management of disconnection. Fault tolerance is now a relatively mature field of computing that can rely on a significant body of theoretical and experimental results. The objective of this work is the analysis and development of a mechanism for fault tolerance for the Ad-Hoc networks. It must support the addition and deletion of dynamic nodes, ensuring the continuation of service despite the presence of different types of failures and the transparency of the process proposed for the users. The rest of the paper is organized as follows: Section 2 describes the Ad-Hoc networks and their characteristics. Sections 3 and 4 present the approach of fault tolerance and related work. Sections 5 define how we use the clustering. Section 6 details our service of fault tolerance. Section 7 gives an evaluation of our propositions. At last, we conclude in Section 8. 


\section{AD-HOC NETWORK}

A mobile Ad-Hoc network (MANET) is a group of mobile nodes forming a temporary network without the aid of any fixed infrastructure or centralized administration [7][15]. The configuration of this network can be static or dynamic. Its life is variable but can be very limited. In addition, it incorporates the specific characteristics of any mobile network standard, among others [2][11]:

- Mobility is the rule rather than the exception, is the essence itself of the network;

- The disconnection: voluntary or involuntary, temporary or permanent;

- Instability in the storage of data: Is it available at all times for backup operations?

- The low-capacity storage media except some hosts such as laptops;

- Low bandwidth (in the present state of technological progress). This factor is closely linked to technological change;

- The low power mobile sites: Batteries are very far from the stability of the energy made available in fixed networks. This factor will play a very important and is closely related to other factors;

- The limited computing power (except for laptops);

- High vulnerability to failures due to the fragility of the environment;

- The high vulnerability to intrusion.

In general, routes between nodes in an ad-hoc network may include multiple hops and, therefore, it is appropriate to call such networks "Ad-Hoc" networks multi-hop. Figure 1 shows an example of mobile Ad-Hoc and communication topology.

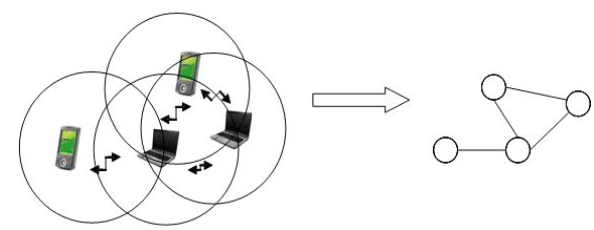

Figure 1 Ad-Hoc network

As shown in Figure, an ad-hoc network may consist of several computer devices such as PDA's, laptops and so on. Each node can communicate directly with other nodes that lie in its radius of transmission. To communicate with nodes that reside around its radius, the node needs to use intermediate nodes to send the message by jumping. MANET can be modeled by a graph $G_{t}=\left(V_{t}, E_{t}\right)$ with $V_{t}$ all network nodes and all connections between these nodes. If $u$ and $v$ belong to $V_{t}$ and $e=(u, v)$ belongs to and then the nodes $u$ and $v$ can communicate directly to the instant $t[11]$.

In mobile environments the terminals are subject to voluntary or involuntary disconnections (Figure 2). The first decided by users for its mobile terminal are justified by the expected return on the cost of communications, energy, service availability and minimizing application approvals induced by unannounced disconnections. The latter are the result of cuts of physical network connections, for example by passing the user in a gray area radio. 


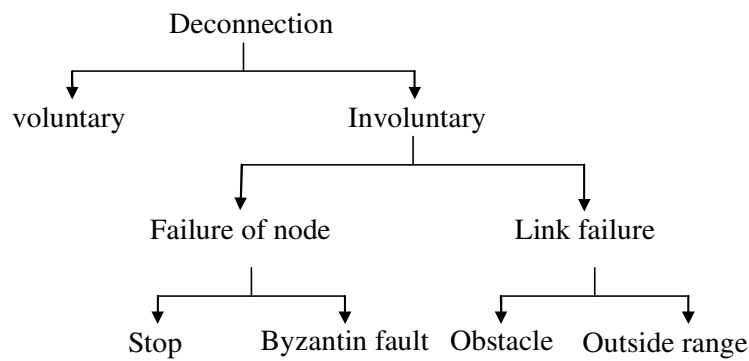

Figure 2. Disconnection in mobile environment

\section{APPROACHES OF FAULT TOLERANCE}

For the Fault tolerance is the ability of a system's performance in spite of faults [7][12]. Other means of dependability can be considered to build secure operating systems (i.e. the elimination, prevention or prediction errors). The purpose of fault tolerance is to increase the reliability of a system. As part of the work in this manuscript, we focus exclusively on fault tolerance to make the availability of data. The requirement of fault tolerance is a key player in distributed systems. On the one hand, fault tolerance is a need brought about by the multiplicity of resources and many other work on distributed systems are designed to ensure the safe operation of these systems is not degraded by the distribution. In addition, fault tolerance may be in itself a motivating factor distribution. Indeed, the fault tolerance can be achieved without redundancy and the distribution of treatments and data on different processors can organize and manage this redundancy. Fault tolerance in such systems is implemented mainly by software, so you can probably expect a degree of continuity means opposite developments in the hardware [3][10].

To tolerate faults, replication is a fundamental technique used in distributed systems. It is to store the same data or service in several nodes. Data or services are often replicated to improve availability, reliability, tolerance to faults, and performance. Replication can also improve data availability and service when the server crashes. Mobility or failure of one node can lead to network partitioning, where the network is divided into disjoint partitions, caused by the possibility of inconsistent data [10][13].

\section{RELATED WORKS}

Hauspie and Buttyan [1][4] have proposed a new metric to detect the partitioning of the network without using GPS. The metric is based on finding a set of paths between a node disjoins client and a server node. A set of paths is a disjoint set of paths that have no common node except the node client and server node. The decision to replicate a service or data is taken when the connection between client and server is getting worse in terms of sociability, bandwidth, delay, etc. Replicating the service or the data on a node that is closest to the client node can increase the quality of the connection between the client and server nodes.

Jorgic et al. [6] have proposed algorithms to detect localized nodes and critical links that could divide the network. A node $u$ is said $k$-critical if the sub graph of neighbors $k$ jumps, which we exclude $u$ and the links leading to it is not related. In a similar detection of critical links, a link $u v$ is $k$-critical sets of neighbors $k$ jumps of $u$ and $v$ (constructed assuming that the link $u v$ does not exist) are disjoint. If a link is critical Overall, it will be $k$-critical whatever $k>1$.

Thanedar et al. [14] have proposed a schema of replication, called the replication ring expansion (Expanding Ring Replication). The data server measures the frequency of requests for each data. If it exceeds a threshold value, he said the data on one or more nodes capable of its 
neighborhood. The capacity function considers parameters such as memory space available, the remaining battery power and processing capacity.

Song et al [9] have proposed new cost-effective wireless access algorithms incorporating a present replicated server allocation algorithm with more keen analysis of the moving patterns of mobile device users. They propose four different algorithms that allocate available replicated servers in the network so as to minimize the communication costs.

\section{MOdel-Based Cluster}

To make our service scalable and to better conserve energy by avoiding communication long range and improve availability. We decided to partition the network into a number of clusters (groups). Clustering is a virtual switching network nodes into groups of geographically close. These groups are called "clusters". They are usually identified by a particular node, for manage the group called "Leader". The leader has the responsibility to manage the replicas, the requests and the propagation of the update. The clustering is particularly suited to Ad-Hoc networks characterized by low mobility. The structure of the cluster architecture is shown in Figure 3.

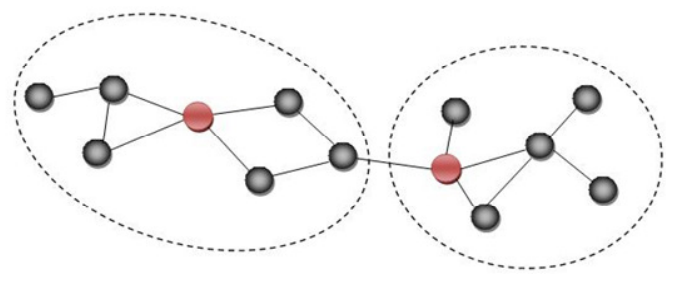

Figure 3. Clustering

Each node in the group communicates regularly with the leader by sending his information: identifier, energy level, neighboring nodes, number of times he stopped, and the directory replicas that exist in this node. It's assumed that each node has a unique address, called the ID_node, a line refers to one given.

We organize all leaders in order to manage replication and messages exchanged between the nodes, and every leader in establishing a directory of different replica in its cluster. To form clusters, we propose a fully distributed algorithm based on the level of energy. In the literature, we find several variants for the election of a leader [12]. We can include: density, number of neighbors, available storage capacity, etc.

To elect a leader we propose the following algorithm:

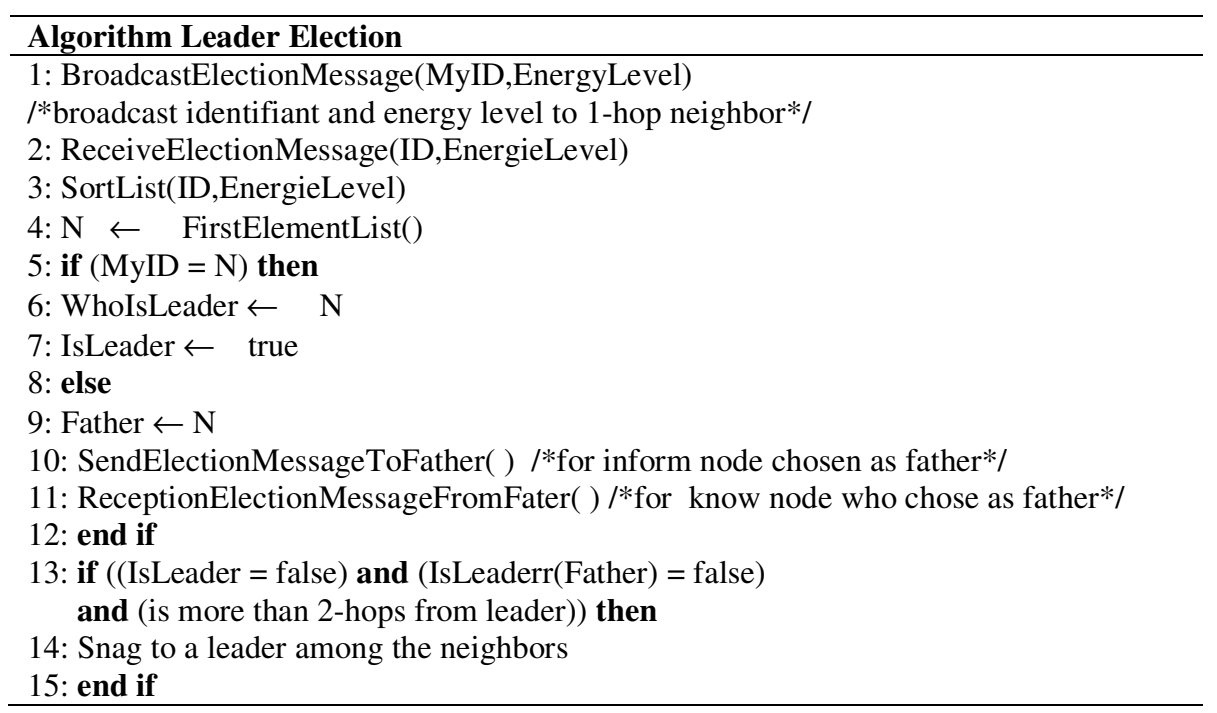




\section{Fault Tolerance Service}

The Ad-Hoc networks where nodes move freely and have their batteries diminish quickly, causing connection failures and frequent node failures. The failure of some links and nodes considered as criticism can divide the network into several partitions. This reduces the availability of data and leads to inconsistent data.

Data replication in Ad-Hoc networks must address the additional problems resulting from the constraints imposed by the environment of Ad-hoc network [4][7]. These problems are:

- Problem partitioning of the network : Partitioning reduces the chances of access to a data because mobile users may not be in the same partition as the node holding the data. Replication of data in the partitions before the occurrence of network partitioning can improve the availability of data. To do so, the replication define the response time of network partitioning may occur and replicate data in advance.

- Problem of energy consumption : In mobile communications, an important parameter is the energy under the battery, in fact the Ad-Hoc networks are autonomous and independent so they do not opportunity to have an infinite energy as it once consumed can not be recovered or reloaded. To increase the availability of data, the replication protocol must replicate a critical data on the nodes that can last for a long time.

Problem scalability : As the network size increases, a request sent by a client node may have to traverse a long path to reach the data, thus increasing the cost and latency of motion. Moreover, the existence of a large number of customers request causes controversy over access channel, which reduces the available bandwidth and increases the access delay of the channel. The replication protocol should be designed so that its performance is not greatly accepted if the number of nodes or the network size increases.

The model proposed for tolerating faults in the Ad-Hoc networks is a model which consists of four sub-services, which are presented in Figure 4.

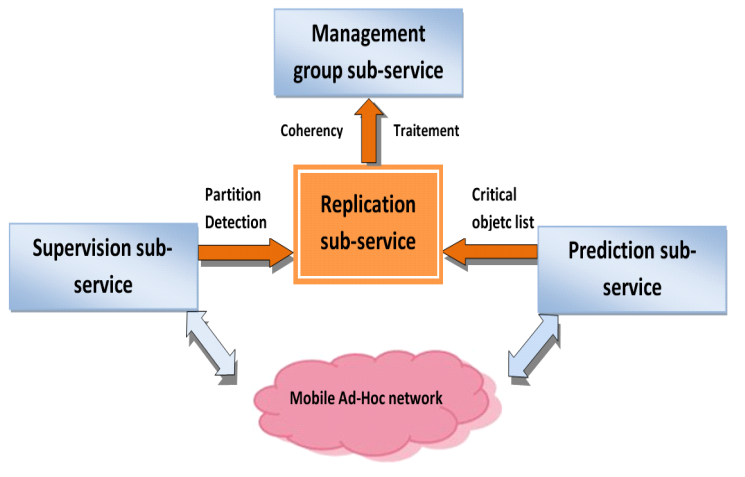

Figure 4. Architecture of fault tolerance service

The model is based on the notion of prediction. The prediction algorithms are aimed at improving the life of a service to ensure that data are always available, for the detection of possible failures or breaches of the connectivity of the network. The mobility of nodes and the limited scope of communication imply the possibility of seeing any possibility of accessing data.

The sub-service prediction predicts a possible failure or disconnection of the network by establishing a list of critical object [4][6]. Each leader can know the current status of all nodes of its group. To assess the criticality, the sub-service can detect several types of items critical energy point of articulation, isthmus and frequent failure or shutdown. To control the energy, 
we classify the level of energy in three statements: High energy, Average energy and Low energy. If an energy level of a node reaches "low energy" the prediction as the class critic node.

In [13], the authors define as the prediction is used as part of a service or client application is in communication with a server application for some time. It is therefore unnecessary to seek a comprehensive disconnections witch may occur throughout the network. Only breaks speakers available on the paths between the client and the server will look. Moreover, it is better to leave the assessment to the client because the server can not afford to carry out measures that can be costly for each of its customers (which may be many). The author therefore proposes to make measurements on the roads or available between server and client at regular intervals. If a weakness is detected, we can prevent two applications which can then adapt their behavior according to their policy of responding to network disruptions.

The sub-service of replication is regarded as a central service of fault tolerance. A basis of the results of the previous service (list of critical objects) a decision of replication is taken. The sub-service which is the previous sub-service will provide prediction that sub-service from the current state of the network a list containing all the nodes and links deemed critical on the basis of this list sub-service replication to better manage the replicas. We define nodes reliable as a node which is not considered by the sub-service prediction as critical and has the highest energy level among the cluster. According to the list of this sub-critical service can be in front of three decisions:

- Do nothing (do not replicate), if the list of critical object is empty.

- Deleting replication (the maximum of replica in cluster is two).

- Replication decision, replication of a base list of critical objects.

The following activity diagram illustrates the decision Replication sub-service. From the list of critical items provided by the sub-service prediction, replication sub-service decides to replicate the data (see Figure 5).

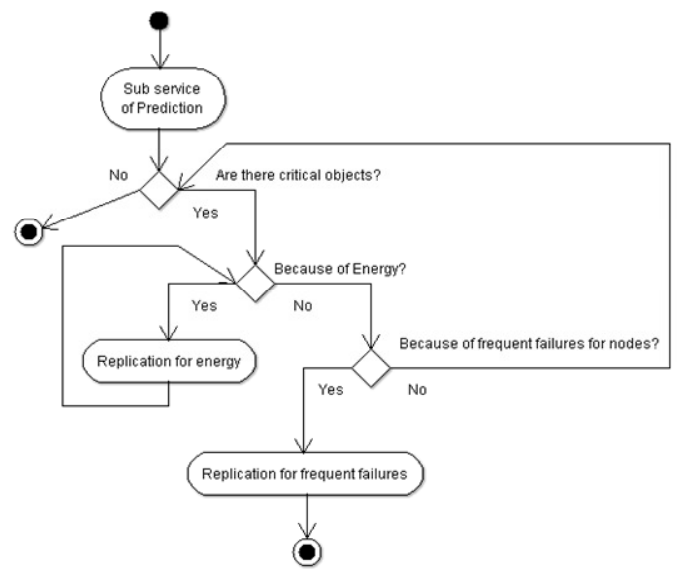

Figure 5. Activity diagram decision replication

To monitor the network, we proposed the use of supervision sub-service. The role of this sub-service is to detect network partitions. Each leader in performing the service for the nodes related to the partition.

The detector of partition maintains the topology of a partition in the network. Disseminating messages of heart beats. When a node sends a reply, it is put in the whole process accessible. Each node $p$ executes the Leader detection algorithm constructs a partition and all nodes accessible to $p$ rated partition $p$. This algorithm runs periodically at each change of network topology. The sub-service of group management maintains the consistency between the replicas. From the viewpoint of data management must provide copies of their mutual consistency, i.e. 
that all copies of data are identical. Without control, the simultaneous execution of requests for access may lead to undesirable phenomena. To improve consistency between replicas, the subservice management group begins with the propagation of the update when a node submits a query and update (write). And when one or more nodes join the cluster the sub-service launches reconciliation.

\section{SiMULATION AND RESULT}

In this section, we study the performance of the proposed service using AdHocFTSim. AdHocFTSim is a simulator for Ad-Hoc network that we developed in java. In the simulator the nodes move according to the waypoint mobility model. In this model, a node randomly selects a location and moves toward it with a constant speed uniformly distributed between zero and a maximum speed Vmax, and then it stays stationary during a pause time before moving to a new random location. Initially, each mobile node has a level of energy represented in percentage. All the simulation, nodes start with full battery.

\section{a. Impact of the range}

In the first simulation, we wanted to determine the impact of the range on the number of requests accepted/lost.

The simulated networks are composed of 50 nodes, 200 requests (reading), 20 data, simulation time 60 seconds, time pause 1 second and mobility speed $3 \mathrm{~m} / \mathrm{s}$. We considered the range from 50 to $200 \mathrm{~m}$ in steps of 50 . The result of this experiment is shown in Figure 6. With a range of $100 \mathrm{~m}$ the network is completely connected (no partition), which is why the number of lost motion whether with or without our approach is zero. Above $150 \mathrm{~m}$ there is a significant increase in the number of lost motion. However with our approach the number of lost motion is reduced. With a range of $50 \mathrm{~m}$, the number of lost motion is very large because there are many partitions in the network.

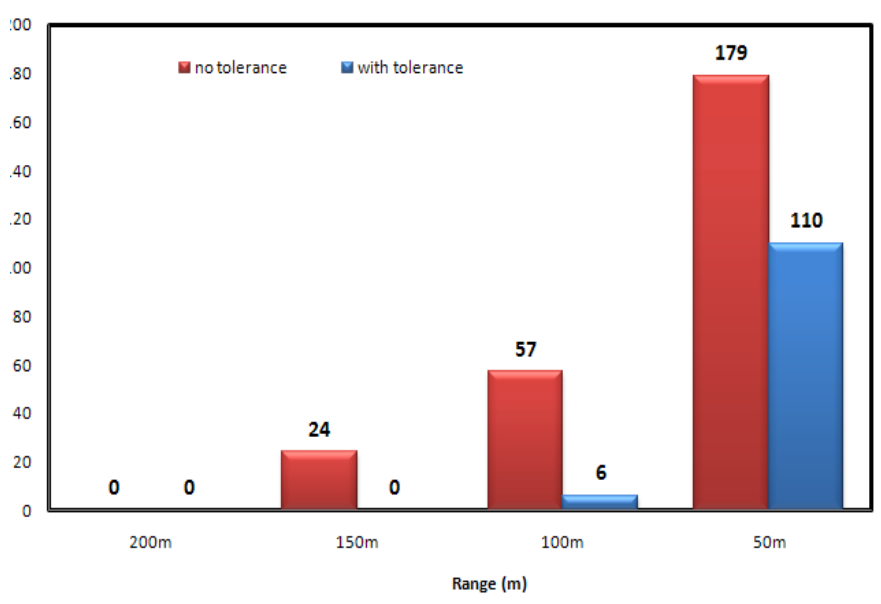

Figure 6. Impact of the range

\section{b. Impact of voluntary fault}

To measure the impact of the number of nodes that voluntarily decide to stop in the network, we proposed a series of simulation with the same parameters of the previous experiment except that this time, we vary the number of stops volunteer in the network from 0 to 8 stops in steps of two. 
The Figure 7 shows the contribution of our approach with fault tolerance to compare to the number of voluntary stops in the network. We note that the number of requests lost with our approach is always zero even increase stops volunteers. This is due to the replication before the nodes stops. While no tolerance, the number of lost requests increases exponentially.

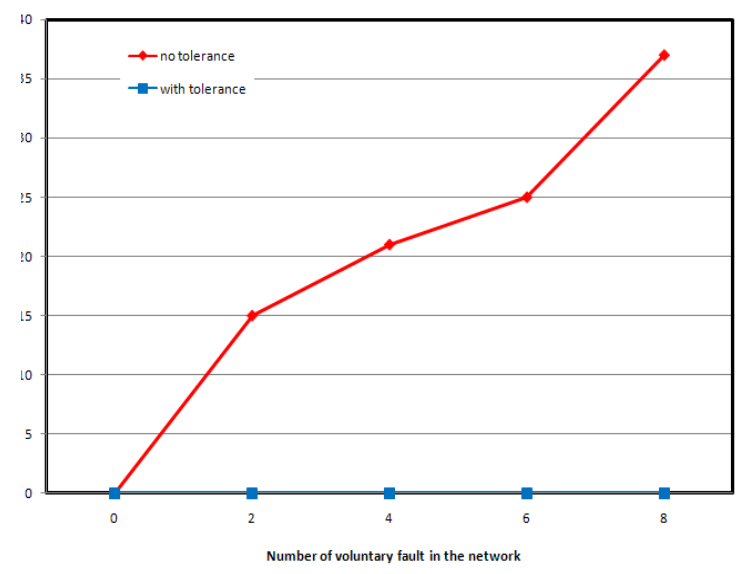

Figure 7. Impact of voluntary fault

\section{c. Impact of number of nodes}

A network is unstable when the pause time is low (less than one second). After several rounds of simulations we found that the network is more stable our service will save more energy.

The second simulation shows the impact of the number of node on the energy consumption. We conducted a simulation with the same parameters of the previous simulation by varying the number of nodes from 100 to 700 nodes in steps of 100 . The simulation results are expressed in Figure 8 .

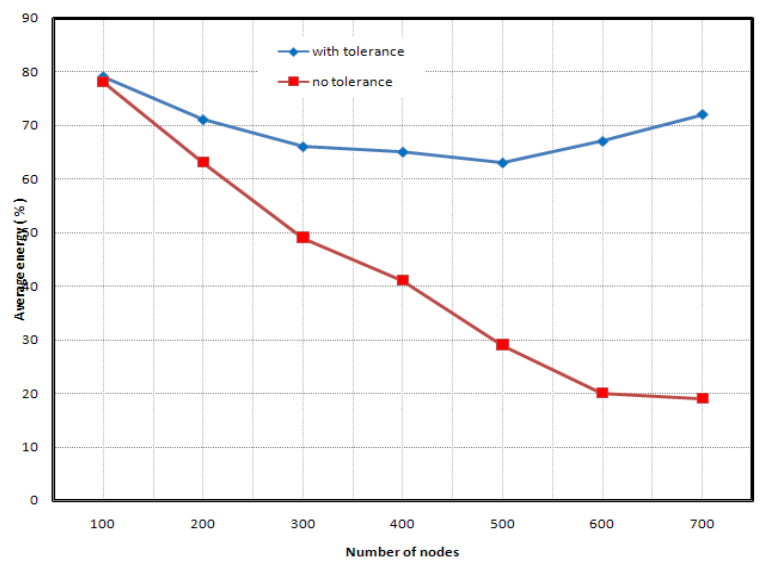

Figure 8. Impact of the number of nodes

It can be seen in Figure that energy consumption with our approach is still low compared to an approach without fault tolerance. This is due to the reduction of long distance communications, which are very costly in energy. We also note that from 500 nodes the conservation of energy is better with our approach (see Figure 9), it confirms the property to scale. 


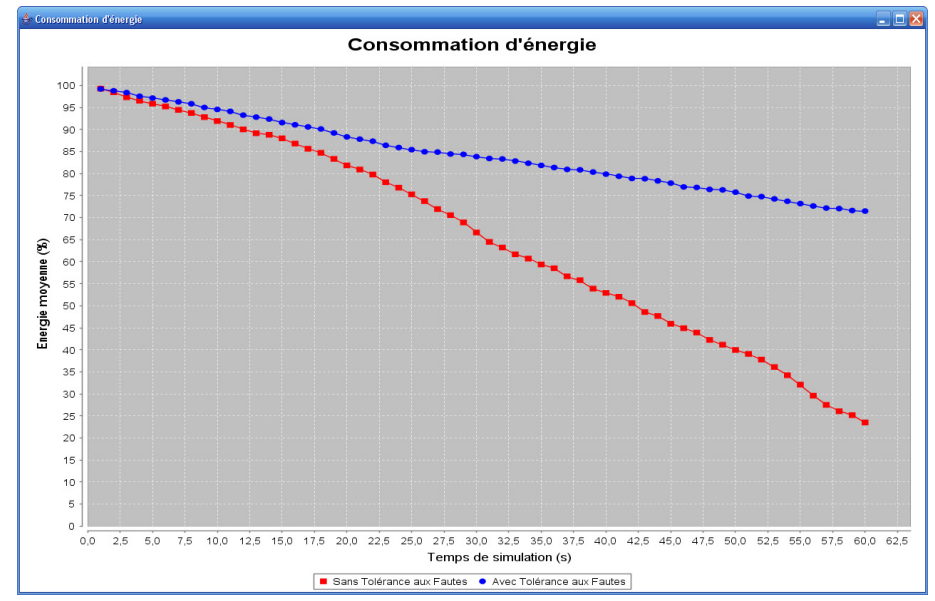

Figure 9. Conservation of energy

\section{d. Impact on the life of the network}

Figure 10 shows the lifetime of nodes in the network. We took the same parameters as the previous simulation but with a number of nodes equal to 300 and a simulation time of $240 \mathrm{~s}$. From this figure, we can see that our proposed with fault tolerance, can conserve energy better than the standard approach, increasing the overall lifetime of the network.

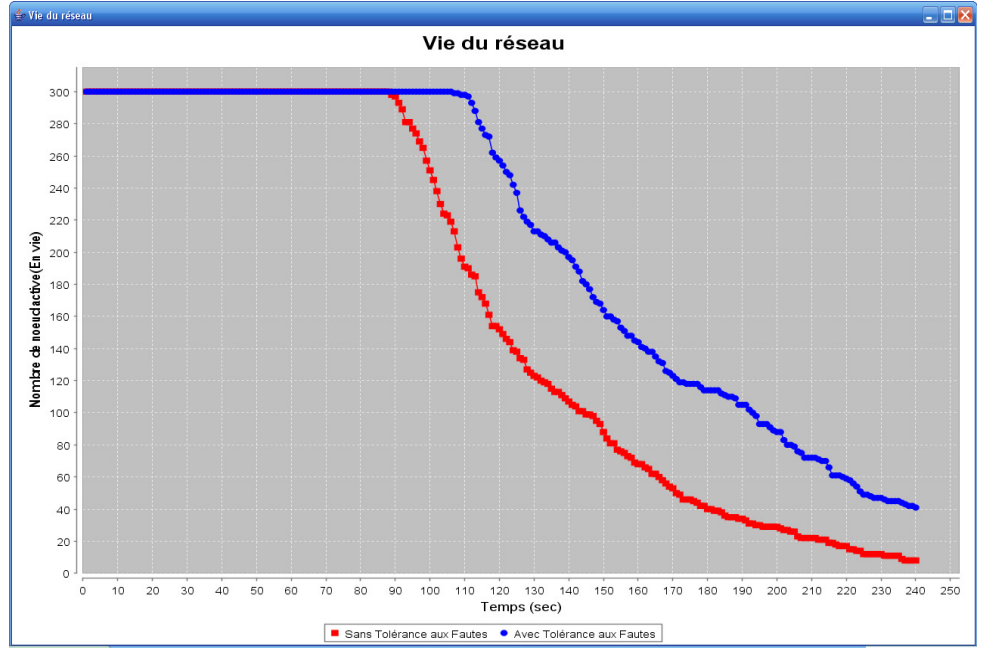

Figure 10 Evolution of Life Network

\section{Conclusion ANd Future Work}

We have given a solution for the management of fault tolerance in the Ad-Hoc networks, combining the functions needed to better availability of data. Our contribution takes into account the characteristics of mobile terminals in order to reduce the consumption of resource is critical (energy), and to minimize the loss of information. The experimental results obtained have shown that our solution allows reducing the number of lost requests, reducing response times to queries, better conserve energy which increases the lifetime of the network and 
supporting the scalability. In the future, we can extend this work by taking into account firstly the consistency of replicas in such a highly dynamic environment, and secondly by incorporating a model of energy to reduce by advantage in energy consumption [5][9].

\section{ACKNOWLEDGEMENTS}

We thank laboratory LIO (Laboratory of computer Science, Faculty of Sciences, Dept. Computer Science of Oran, Algeria) for the means which it placed at our disposal.

\section{REFERENCES}

[1] Buttyan, L. and Hubaux, J.-P. (2000) 'Enforcing service availability in mobile ad-hoc WANs'. In Proc. of the 1st ACM International Symposium on Mobile Ad Hoc Networking and Computing (MobiHoc 2000). Boston, Massachusetts: ACM, 2000, pp. 87-96.

[2] Goncalves, B., Mitton, N. and Guérin-Lassous, I. (2006) 'Comparison of two Self-Organization and Hierarchical Routing Protocols for Ad Hoc Networks'. In Second International Conference on Mobile Ad Hoc and Sensor Networks (MSN), Hong-Kong, China, December.

[3] Hamdy, M. and Konig-Ries, B. (2008), 'A service distribution protocol for mobile ad hoc networks', ICPS '08: Proceedings of the 5th international conference on Pervasive services, Sorrento, Italy, pages. 141-146.

[4] Hauspie, M. and Simplot, D. and Carle, J. (2003), 'Partition Detection in Mobile Ad-hoc Networks', In Proceeding of the 2nd Mediterranean Workshop on Ad Hoc Networks (Med-Hoc-Net 2003), Mahdia, Tunisia.

[5] Ito, S. and Yoshigoe, K. (2009), 'Performance Evaluation of Consumed Energy-Type-Aware Routing (CETAR) For Wireless Sensor Networks', International Journal of Wireless \& Mobile Networks (IJWMN), Vol. 1, No. 2, November 2009, pp. 90-101.

[6] Jorgic, M. and Stojmenovic, I. and Hauspie M. and Simplot-Ryl, D. (2004), 'Localized algorithms for detection of critical nodes and links for connectivity in ad hoc networks'. In Proceeding of the Third Annual Mediterranean Ad Hoc Networking Workshop, Med-Hoc-Net, June, Bodrum, Turkey.

[7] Michiardi, P. and Molva, R. (2004), 'Mobile Ad Hoc Network', Wiley-IEEE Press, ch.12: Ad Hoc Network Security, pp. 329-354.

[8] Mitton, N., Busson, A. and Fleury, E. (2006) 'Efficient broadcasting in self-organizing sensor networks', International Journal of Distributed Sensor Networks (IJDSN), Vol. 2, No 2, pp. 161-187.

[9] Nitnaware, D. and Verma A. (2010), 'Energy Constraint Node Cache Based Routing Protocol For AdHoc Network', International Journal of Wireless \& Mobile Networks (IJWMN), Vol. 2, No. 1, February 2010, , pp.77-86.

[10] Padmanabhan, P. and Gruenwald, L. and Vallur, A. and Atiquzzaman, M. (2008), 'A survey of data replication techniques for mobile ad hoc network databases', The VLDB Journal, Vol.17, No. 5, pp. 1143-1164.

[11] Perkins, C. (2001), 'Ad Hoc Networking'. Addison-Wesley.

[12] Randell, B., Avizienis, A., Laprie, J-C. and Landwehr, C. (2004) 'Basic concepts and taxonomy of dependable and secure computing', IEEE Transactions on Dependable and Secure Computing, Vol.1, No. 1, pp.11-33.

[13] Simplot, D. and Hauspie, M. and Carle, J. (2002). 'Replication decision algorithm based on link evaluation for services in MANET'. Technical Report 2002-05, IRCICA/LIFL, Univ. Lille 1. France.

[14] Thanedar, V. and Almeroth, K. C. and Belding-Royer E. M. (2004), 'A Lightweight Content Replication Scheme for Mobile Ad Hoc Environments', In Proceeding NETWORKING (Networking Technologies, Services, and Protocols; Performance of Computer and Communication Networks; 
Mobile and Wireless Communication), Third International IFIP-TC6, Athens, Greece, May 9-14, LNCS Volume $=3042$. pages $=125-136$

[15] Upadhayaya, S. and Gandhi, C. (2009), Quality of Service Routing in Mobile Ad Hoc Networks Using Location anf Energy Parameters, International Journal of Wireless \& Mobile Networks (IJWMN), Vol 1, No 2, November 2009, pp. 138-147.

\section{Authors Biography}

Ghalem Belalem graduated from Department of Computer Science, Faculty of Sciences, University of Oran, Algeria, where he received $\mathrm{PhD}$ degree in computer science in 2007. He is now a research fellow of management of replicas in data replicas in AdHoc network, data grid and cloud computing. His current research interests are distributed systems, grid computing and data grid placement of replicas and consistency in mobile environment.

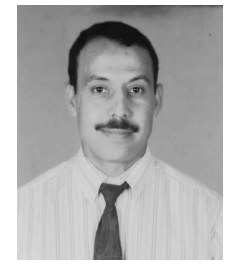

Ali Cherif Brakeche is graduate from University of Oran (Es Senia), Algeria, where he received his Master's degree in Computer Science (June 2009) from Faculty of Sciences, University of Oran, Algeria. His research interests are replication and consistency strategies, wireless communications and networking, with a current focus on throughput improvement of multihop wireless networks.

Abderahmann Benaissa is graduate from University of Oran (Es Senia), Algeria, where he received her Master's degree in Computer Science (June 2009) from Faculty of Sciences, University of Oran, Algeria. His research interests are replication and consistency strategies, data grid and wireless grid.

Esma Insaf Djebbar is graduate from University of Oran (Es Senia), Algeria, where she received her Master's degree in Computer Science (June 2008) from Faculty of Sciences, University of Oran, Algeria. Her research interests are replication and consistency strategies, fault tolerant, wireless network, data grid and Cloud Computing. She is a Ph.D. student at Oran University (Es Senia), Algeria since 2010. 